\title{
PING Gamma Ray and Neutron Measurements of a Meter-Sized Carbonaceous Asteroid Analog
}

\author{
J. Bodnarik ${ }^{1,2}$, D. Burger2, L. Evans ${ }^{1,3}$, S. Floyd ${ }^{1}$, L. Lim ${ }^{1}$, T. McClanahan1, \\ M. Namkung ${ }^{1}$, S. Nowicki ${ }^{1,4}$, A. Parsons ${ }^{1}$, J. Schweitzer ${ }^{5}$, R. Starr ${ }^{1,6}$, J. Trombka ${ }^{1}$ \\ ${ }^{1}$ NASA Goddard Space Flight Center, ${ }^{2}$ Vanderbilt University, ${ }^{3}$ Computer Sciences Corporation, \\ ${ }^{4}$ Univeristy of Michigan, 5 University of Connecticut, ${ }^{6}$ Catholic University of America
}

\section{SUMMARY}

Determining the elemental composition of carbonaceous (spectral type C) asteroids is still one of the basic problems when studying these objects. The only main source of elemental composition information for asteroids is from their optical, NIR and IR properties, which include their spectral reflectance characteristics, albedo, polarization, and the comparison of optical spectroscopy with meteorite groups corresponding to asteroids of every spectral type. Unfortunately, these sources reflect observations from widely contrasting spatial scales that presently yield a void in the continuum of microscopic and macroscopic evidence, a lack of in situ measurement confirmation, and require deeper sensing techniques to discern the nature of these asteroids. The Probing In situ with Neutrons and Gamma rays (PING) instrument is ideally suited to address this problem because it can be used to determine the bulk elemental composition, $\mathrm{H}$ and $\mathrm{C}$ content, the average atomic weight and density of the surface and subsurface layers of Ctype asteroids, and can provide measurements used to determine the difference between and distinguish between different types of asteroids.

Gamma ray and neutron instrumentation techniques have been used extensively in oil and scientific well logging applications to determine the bulk elemental composition of subsurface geology [1]. The current gamma ray and neutron instrumentation development effort at NASA Goddard Space Flight Center's Astrochemistry Laboratory aims to extend the use of these techniques to probe the surface and subsurface of carbonaceous (spectral type C) asteroids in situ without the need to drill. We are currently developing the PING instrument that combines gamma ray and neutron detectors with a $14 \mathrm{MeV}$ pulsed neutron generator to determine the in situ bulk elemental abundances and geochemistry of C-type asteroids with a spatial resolution of $1 \mathrm{~m}$ down to depths of tens of $\mathrm{cm}$ to $1 \mathrm{~m}$ [2]. One aspect of the current work includes experimentally testing and optimizing PING on a known meter-sized Columbia River basalt $\mathrm{C}$-type asteroid analog sample that has a similar composition and the same neutron response as that of a C-type asteroid. An important part of this effort focuses on utilizing timing measurements to isolate gamma rays produced by neutron inelastic scattering, neutron capture and delayed activation processes. Separating the gamma ray spectra by nuclear processes results in higher precision and sensitivity elemental composition measurements. Using gated data acquisition techniques allows for the unambiguous identification of gamma ray lines from different isotopes and nuclear processes, especially in situations when limited detector resolution results in overlapping gamma ray lines that cannot be individually resolved. 
Another aspect of this work includes conducting extensive Monte Carlo N-Particle extend (MCNPX) computer modeling runs to determine the appropriate composition and configuration of the basalt analog sample which closely approximates the neutron response for that of a C-type asteroid. To insure that the neutron response for the basalt sample is like that of a C-type asteroid, the key elements are that the thermal and epithermal neutron fluxes, as a function of depth beneath the surface, need to closely approximate those of a C-type asteroid. MCNPX modeling results for the basalt analog sample are in excellent agreement with the C-type asteroid CI1 chondrite composition model to within $1 \%$ error. This agreement is especially important from the surface to a depth of about $30-35 \mathrm{~cm}$, since the majority of the gamma rays produced through nuclear interaction processes will be coming from this volume, as shown in the Figures $1 \& 2$.
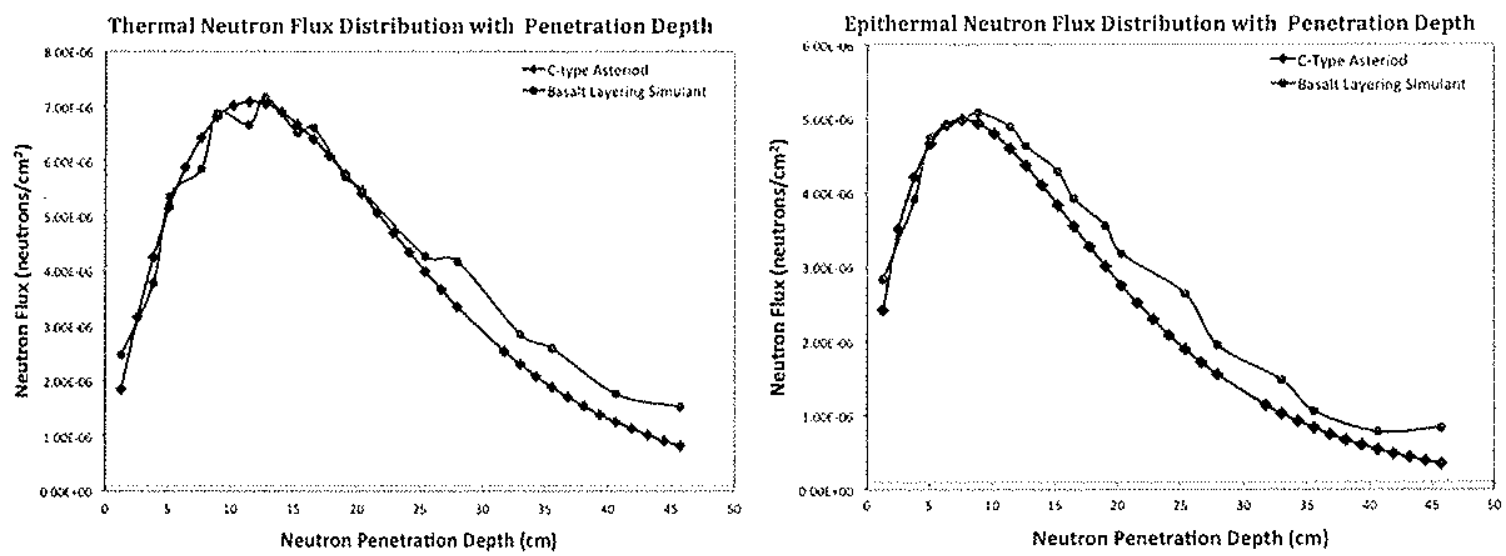

Figures $1 \& 2$. Graphs of the thermal and epithermal neutron flux distribution as a function of neutron penetration depth for both the C-type asteroid (blue) and the basalt layering simulant (red).

In this paper, we will present the PING basalt layering experimental data, taken at the test facility at NASA Goddard Space Flight Center using the time tagged event-by-event data analysis technique, compared to our MCNPX computer simulation results for the C-type asteroid and basalt layering simulant models. Comparison of these data will show the advantages, validity, and measurement sensitivity of PING's nuclear interrogation methods to obtain more precise and sensitive in situ bulk elemental composition and density measurements of the subsurface of asteroids.

\section{REFERENCES}

I. J. A. Grau, J. S. Schweitzer, J. K. Draxler, J. Lauterjung, "Elemental Logging in the KTB Pilot Hole. 1: NaI-based Spectrometry", the International journal of radiation applications and instrumentation. Part E. Nuclear geophysics. Industrial radiation and radioisotope measurement applications. Conference, Raleigh NC, 1993, vol. 7, no. 2, pp. 173-187.

2. A. Parsons, J. Bodnarik, L. Evans, S. Floyd, L. Lim, T. McClanahan, M. Namkung, J. Schweitzer, R. Starr, J. Trombka, "Planetary Geochemistry Using Active Neutron and Gamma Ray Instrumentation", 41 st Lunar and Planetary Science Conference (2010), vol. 41, pp. 2553. 